\title{
Efecto del clima en los anillos de crecimiento de Araucaria araucana en el norte de la Patagonia Argentina
}

\author{
M. Hadad ${ }^{12^{*}}$ \\ (1) CIGEOBIO-CONICET, (FCEFyN-UNSJ), Av. José Ignacio de la Rosa 590 oeste. Rivadavia. CP: J5402DCS, San Juan, Argentina. \\ (2) Departamento de Dendrocronología e Historia Ambiental. IANIGLA, CCT CONICET-Mendoza, CC 330, Av. Ruiz Leal s/n, Mendoza, Argentina.
}

*Autor de correspondencia: M. Hadad [mhadad@mendoza-conicet.gob.ar]

> Recibido el 25 de julio de 2014 - Aceptado el 24 de septiembre de 2014

Hadad, M. 2014. Efecto del clima en los anillos de crecimiento de Araucaria araucana en el norte de la Patagonia Argentina. Ecosistemas

23(3): 109- 111. Doi.: 10.7818/ECOS.2014.23-3.15

Araucaria araucana (Molina) K. Koch (Pehuén), es una conífera dioica, endémica de los bosques templados del centro-sur de Argentina y Chile, distribuida entre 900 y $1800 \mathrm{~m}$ de altitud y entre los $37^{\circ} 20^{\prime}$ y $40^{\circ} 20^{\prime}$ de latitud sur. Los bosques de Araucaria en contacto con la estepa patagónica (xerófilos) tienden a ser puros y diseminados en grupos aislados, conformando bosques fragmentados. El clima en el extremo sur de América del Sur (considerado entre los paralelos $33^{\circ}-55^{\circ} \mathrm{S}$ ) muestra patrones de variación espacial y temporal que pueden ser explicados en parte por la influencia atmosférica, topográfica y oceánica (Roig y Villalba 2008). En este sentido, las anomalías de las precipitaciones y temperaturas asociadas con la ocurrencia del fenómeno El Niño y su contraparte La Niña, son la principal fuente de variabilidad interanual en la mayor parte de América del Sur (Garreaud et al. 2009). Aspectos de esta variabilidad climática en latitudes medias y altas de América del Sur son también explicadas por el Modo Anular del Hemisferio Sur (SAM), índice que representa $\sim 35 \%$ del total de la variabilidad en la circulación atmosférica extra-tropical del Hemisferio Sur (Marshall 2007).

Los anillos de crecimiento de las plantas leñosas han demostrado ser un efectivo indicador biológico de las condiciones ambientales que inciden sobre el crecimiento de las plantas, en particular el clima (Fritts 1976). Algunos estudios ecofisiológicos demuestran la relación ambiente-crecimiento y sugieren que ésta puede ser dependiente de la edad y del sexo (ej. Rozas et al. 2009). Comprender estos posibles mecanismos de variabilidad resulta importante, para observar si la sensibilidad del crecimiento a la variación climática tiene diferente expresión según el sexo o la edad dependiendo de la especie.

En esta tesis fueron utilizadas cronologías de anillos de árboles en bosques xéricos de $A$. araucana localizados al norte de la Patagonia. El objetivo fue identificar la intensidad en la respuesta al clima en unidades de bosques maduros, con representación de diversas clases de edad y sexo. Estos estudios resultan relevantes en la interpretación de estrategias del crecimiento de los árboles, desarrollo de los bosques y en el diseño de estrategias de conservación de estos ecosistemas de bosques de Araucaria araucana en ambientes patagónicos xéricos.
En 5 unidades de bosque xérico de $A$. araucana (Tabla 1 y Fig. 1), se tomaron dos testigos de madera, con barrenos de Pressler, por árbol de forma perpendicular al fuste y a la altura estándar de pecho $(1.30 \mathrm{~m})$. En laboratorio, las muestras fueren secadas, montadas y encoladas sobre soportes de madera y lijadas con lijas de progresivo número de granulometría, a fin de poder resaltar la estructura anatómica del límite de los anillos de crecimiento anuales. El control de calidad de las mediciones de las series del ancho de los anillos fue controlado estadísticamente mediante el empleo del programa COFECHA (Holmes 1983). Los índices de ancho de anillos de árboles se calcularon utilizando el programa de computación ARSTAN 4.0c (Cook 1985). Mediante este programa, las mediciones originales de ancho de anillos fueron estandarizadas empleando una función tipo spline cúbico suavizado con frecuencia de $50 \%$ de corte y período de respuesta de 50 años. Se construyó una cronología regional con todos los árboles femeninos y otra con todos los masculinos. Además se construyeron cronologías para cada clase de edad, correspondientes a árboles jóvenes (<120 años), maduros (121-275 años) y adultos (>276 años).

El coeficiente de correlación de Pearson se calculó comparando la versión residual de cada cronología con los valores mensuales de las variables climáticas de temperatura media mensual, precipitación mensual, El Niño y SAM. Para analizar el vínculo entre el crecimiento de $A$. araucana y datos de eventos El Niño, se utilizó la anomalía de temperatura del mar (SST) para la región 3.4 (en adelante, EI Niño 3.4) (East Central Tropical Pacific SST - 5N-5S; $170-120 \mathrm{~W})$ y para el periodo 1948-2008. Además, el crecimiento de $A$. araucana fue comparado con el índice SAM, el cual está basado en la proyección diaria de las anomalías de altura geopotencial de $700 \mathrm{hPa}$ al sur de los $20^{\circ}$ y para el periodo de 1979-2008. Los datos de EI Niño 3.4 y SAM fueron obtenidos de la Administración Nacional del Océano y la Atmósfera (NOAA).

\section{Resultados y Conclusiones generales}

La comparación del clima con el crecimiento definido por clases de edad, identificó que los árboles jóvenes tienen una relación negativa y significativa $(P<0.05)$ entre el ancho del anillo y la temperatura correspondiente al periodo de crecimiento actual (Fig. 2). 
Tabla 1. Localización de los cinco sitios de estudio y número de árboles/radios utilizados.

\begin{tabular}{|c|c|c|c|c|c|}
\hline Sitios & Código & $\begin{array}{c}\text { Número } \\
\text { de árboles/radios }\end{array}$ & Latitud S & Longitud W & Altitud (m) \\
\hline Río Agrio & (RA) & $78 / 144$ & $37^{\circ} 50^{\prime}$ & $70^{\circ} 58^{\prime}$ & 1594 \\
\hline Primeros Pinos & (PP) & $44 / 82$ & $38^{\circ} 52^{\prime}$ & $70^{\circ} 34^{\prime}$ & 1628 \\
\hline Río Kilca & (RK) & $32 / 58$ & $38^{\circ} 53^{\prime}$ & $70^{\circ} 50^{\prime}$ & 1442 \\
\hline
\end{tabular}

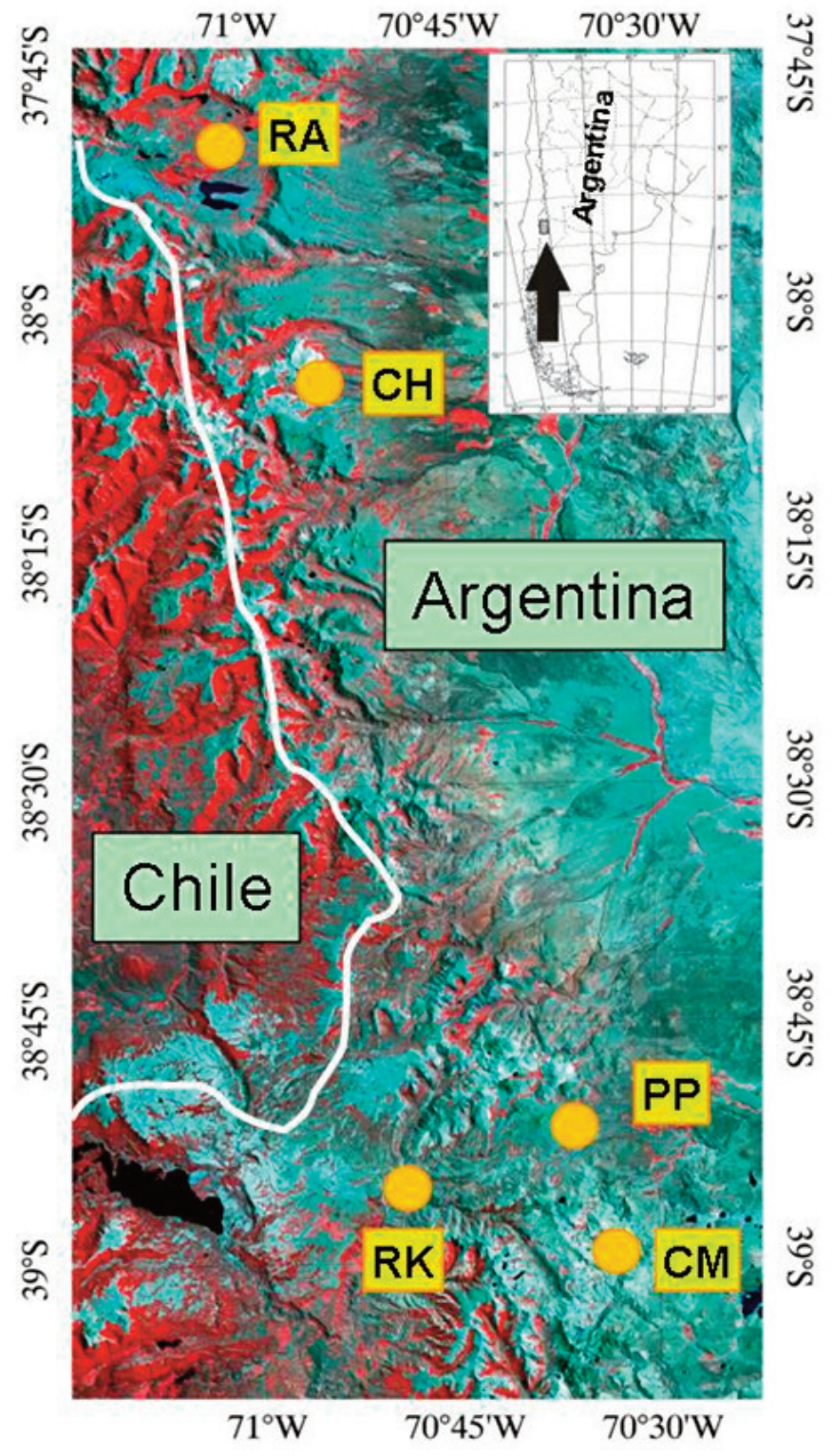

Figura 1. Mapa de los sitios de los bosques de Araucaria araucana, en el norte de la Patagonia. Ver códigos en la Tabla 1.

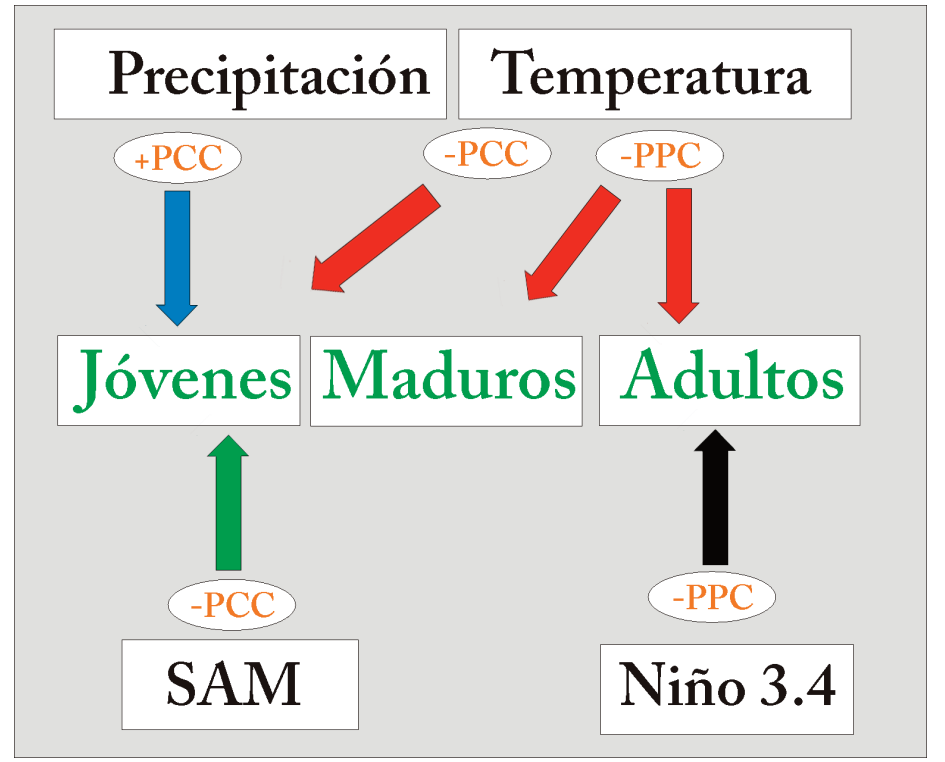

Figura 2. Esquema sintético de las principales tendencias de crecimiento de las clases de edades de Araucaria araucana. Los signos (-) y (+) representan correlaciones significativas negativas y positivas, respectivamente, con un nivel de significancia menores de $P<0.05$. PPC: periodo previo de crecimiento; PCC: periodo corriente de crecimiento. SAM (por sus siglas en inglés): Modo Anular del Sur.

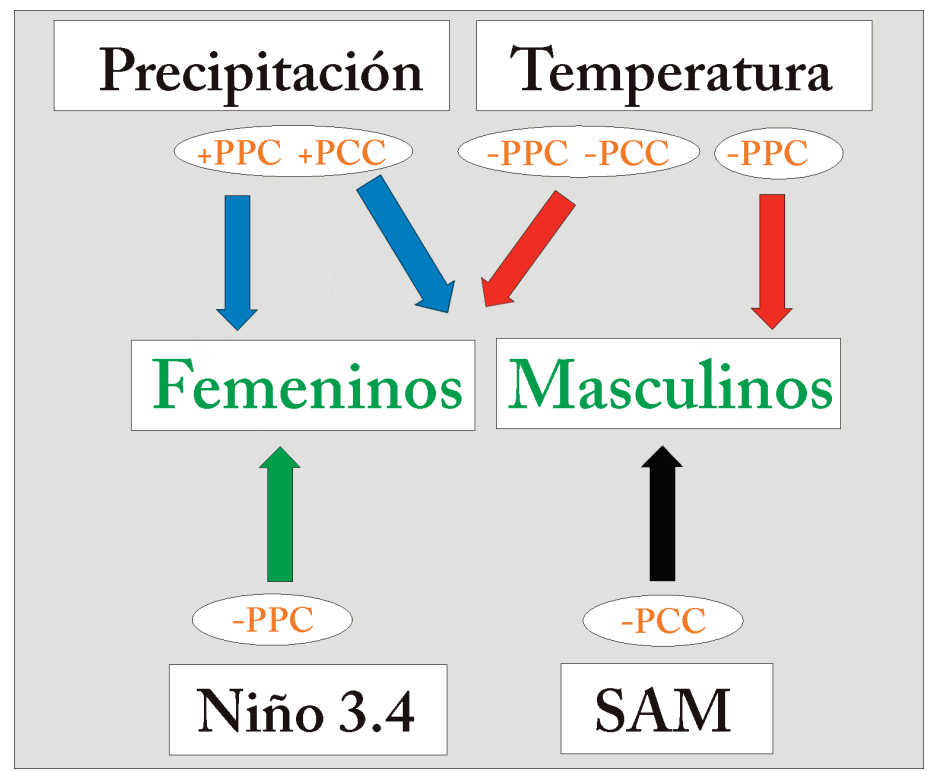

Figura 3. Esquema sintético de las principales tendencias de crecimiento de los árboles masculinos y femeninos de Araucaria araucana. Los signos $(-)$ y (+) representan correlaciones significativas negativas y positivas, respectivamente, con un nivel de significancia de $P<0.05$. PPC; PCC; SAM ver la Figura 2 para su descripción. 
Para los árboles de clases de edad maduros y adultos, la correlación fue negativa y significativa $(P<0.05)$ respecto de las condiciones de temperatura durante el verano previo al crecimiento. En relación a la precipitación, se halló que el crecimiento de los árboles jóvenes presentaba una correlación positiva y significativa $(P<0.05)$ (Fig. 2). Al comparar estos crecimientos con el índice SAM, se observó que los árboles jóvenes presentaban los mayores valores de correlación negativa y significativa $(P<0.05)$ durante el período de crecimiento actual, respecto de las demás clases de edad (Fig. 2). Al comparar el crecimiento con el El Niño 3.4, se observó que los árboles adultos se mostraron más sensibles a la relación con esta variable, especialmente durante los meses previos al crecimiento (Fig. 2). Estos resultados mostrarían que los árboles jóvenes contienen un componente de variabilidad en el ancho de sus anillos de crecimiento que, por un lado, facilitaría la diferenciación de los árboles por su edad, y por otro, a que estos componentes de variabilidad podrían vincularse a particulares respuestas al clima como estrategia de desarrollo durante su etapa juvenil. En este sentido, se conoce que una transición del estado juvenil al estado maduro y reproductivo, incluye no solamente aspectos relacionados a estímulos abióticos, sino que también se manifiestan aspectos relacionados a la fisiología del árbol. En este sentido, la diferencial sensibilidad al clima encontrada en los anillos de crecimiento de $A$. araucana, podría encontrar explicaciones en limitantes estructurales y funcionales en relación a la edad biológica y las oportunidades de crecimiento de esta especie.

Al comparar el clima con cronologías de ancho de anillos de araucaria, pero considerando en este caso series de árboles masculinos y femeninos (Fig. 3). Los árboles femeninos presentaron correlaciones negativas y significativas $(P<0.05)$ durante el periodo corriente y previo de crecimiento, mientras que los árboles masculinos presentaron un correlación negativa y significativa $(P<0.05)$ durante el periodo previo de crecimiento. Las correlaciones del crecimiento de los árboles masculinos y femeninos con la precipitación mensual para el periodo corriente y previo de crecimiento, experimentaron correlaciones significativas $(P<0.05)$ y positivas, siendo los árboles masculinos los que alcanzaron mayor valor de correlación (Fig. 3). Para el caso de la comparación entre el crecimiento y el Índice SAM, los árboles masculinos, en general, tuvieron una mayor sensibilidad durante el periodo corriente de crecimiento (Fig. 3). Al comparar el crecimiento de ambos sexos en $A$. araucana con El Niño 3.4, las correlaciones alcanzadas fueron mayores para los árboles femeninos siendo negativas y significativas $(P<0.05)$ (Fig. 3). De todas estas comparaciones establecidas, surge la hipótesis de que el crecimiento tanto de árboles femeninos como masculinos, podría manifestar sensibilidades diferentes a las variaciones del clima en función de estrategias en el aprovechamiento de los recursos del ambiente.
Se puede concluir que tanto la edad como la característica dioica en araucaria, son variables que se expresan diferencialmente tanto en el crecimiento como en la intensidad de su relación con el clima. Considerando que el siglo XX ha experimentado incrementos en temperatura del aire y disminuciones en la precipitación para amplias zonas de Patagonia (Roig y Villalba 2008, Mundo et al. 2012), y que se pronostica una intensificación de estos fenómenos en el futuro (IPCC 2013), sería esperable condiciones de mayor severidad al crecimiento de los bosques de $A$. araucana. Por ello, el conocimiento aquí presentado expande nuestra frontera del conocimiento sobre la ecología de esta especie, lo que facilitaría generar políticas de manejo y conservación para esta relevante especie forestal en Patagonia.

\section{Agradecimientos}

El autor agradece al CONICET por la beca otorgada para la realización de la Tesis Doctoral. Además agradezco también a Roig. F; Martínez-Carretero, E.; Duplancic, A.; Barrio, E. y Londero S. por la asistencia en el campo y laboratorio. Este trabajo fue apoyado por la Agencia Nacional de Promoción Científica y Técnica (ANPCyT) de Argentina (2010/2679).

\section{Referencias}

Cook, E. 1985. A time series analysis approach to tree ring standardization. Ph.D. dissertation, University of Arizona, Tucson, AZ, Estados Unidos.

Fritts, H. 1976. Tree-Rings and Climate. Academic press. Londres, Reino Unido.

Garreaud, R.D., Vuille, M., Compagnucci, R., Marengo, J. 2009. Presentday South American Climate. Palaeogeography, Palaeoclimatology, Palaeoecology 281: 180-195.

Holmes, R. 1983. Computer-assisted quality control in tree-ring dating and measurement. Tree Ring Bulletin 43: 69-75.

IPCC 2013. Climate Change 2013: The Physical Science Basis. Contribution of Working Group I to the Fifth Assessment Report of the Intergovernmental Panel on Climate Change. Stocker, T.F., D. Qin, G.-K. Plattner, M. Tignor, S. K. Allen, J. Boschung, A. Nauels, Y. Xia, V. Bex and P.M. Midgley, (eds.). Cambridge University Press, Cambridge y Nueva York, Reino Unido y Estados Unidos.

Marshall, G. 2007. Half-century seasonal relationships between the Southern annular mode and antartic temperatures. International Journal of Climatology 27: 373-383.

Mundo, A., Roig Juñent, F.A., Villalba, R., Kitzberger, T., Barrera, M. 2012. Araucaria araucana tree-ring chronologies in Argentina: spatial variations and climate influences. Trees. Structure and Function. 26: 443-458.

Roig, F., Villalba, R. 2008. Understanding climate from patagonian tree rings. En: Rabassa, J. (ed.). The late Cenozoic of Patagonia and Tierra del Fuego. Developments in Quaternary Sciences, 11. (Series Ed. Van Der Meer), pp. 411-438. Elsevier. Amsterdam, Países Bajos.

Rozas, V., DeSoto, L., Olano, J., 2009. Sex-specific, age-dependent sensitivity of tree-ring growth to climate in the dioecious tree Juniperus thurifera. New Phytologist 182: 687-697.

\section{MARTIN ARIEL HADAD}

\section{Análisis florístico-dendrocronológico de los bosques xéricos de Araucaria araucana (Araucariaceae) en el contacto con la estepa patagónica en Neuquén, Argentina}

\section{Tesis Doctoral}

Facultad de Ciencias Exactas, Físicas y Naturales. Universidad Nacional de Córdoba. Argentina.

22 de Marzo, 2013

Director: Dr. Roig Juñent, Fidel Alejandro

Co-director: Dr. Martínez Carretero, Eduardo

\section{$\underline{\text { Publicaciones resultantes de la tesis }}$}

Hadad, M.A., Roig Juñent, F.A., Boninsegna, J.A., Patón, D. 2014. Age effects on the climatic signal in Araucaria araucana from xeric sites in Patagonia, Argentina. Plant Ecology and Diversity Doi: 10.1080/17550874.2014.980350 